\title{
Effect of Subthreshold Slope on the Sensitivity of Nanoribbon Sensors
}

\author{
K. Sun ${ }^{\mathrm{a} \dagger}$, I. Zeimpekis ${ }^{\mathrm{a} \dagger}$, C. Hu ${ }^{\mathrm{a}}$, N. M. J. Ditshego ${ }^{\mathrm{a}}$, O. Thomas ${ }^{\mathrm{b}}$, M. R. R. de Planque ${ }^{\mathrm{a}}$, H. M. H. \\ Chonga , H. Morgan ${ }^{a}$ and P. Ashburna
}

a. Zepler Institute, Electronics \& Computer Science, University of Southampton, Southampton, SO17 1BJ, UK.

b. Oxford Instruments Plasma Technology, Yatton, Bristol, BS49 4AP, UK.

$\uparrow$ These authors have contributed equally.

Email: ks5@ecs.soton.ac.uk, izk@ecs.soton.ac.uk

\begin{abstract}
In this work, we investigate how the sensitivity of a nanowire or nanoribbon sensor is influenced by the subthreshold slope of the sensing transistor. Polysilicon nanoribbon sensors are fabricated with a wide range of subthreshold slopes and the sensitivity is characterized using $\mathrm{pH}$ measurements. It is shown that there is a strong relationship between the sensitivity and the device subthreshold slope. The sensitivity is characterized using the current sensitivity per $\mathrm{pH}$, which is shown to increase from $1.2 \% / \mathrm{pH}$ to $33.6 \% / \mathrm{pH}$ as the subthreshold slope improves from $6.2 \mathrm{~V} / \mathrm{dec}$ to $0.23 \mathrm{~V} / \mathrm{dec}$ respectively. We propose a model that relates current sensitivity per $\mathrm{pH}$ to the subthreshold slope of the sensing transistor. The model shows that sensitivity is determined only on the subthreshold slope of the sensing transistor and the choice of gate insulator. The model fully explains the values of current sensitivity per $\mathrm{pH}$ for the broad range of subthreshold slopes obtained in our fabricated nanoribbon devices. It is also able to explain values of sensitivity reported in the literature, which range from $2.5 \% / \mathrm{pH}$ to $650 \% / \mathrm{pH}$ for a variety of nanoribbon and nanowire sensors. Furthermore, it shows that aggressive device scaling is not the key to high sensitivity. For the first time, a figure-of-merit is proposed to compare the performance of nanoscale field effect transistor sensors fabricated using different materials and technologies.
\end{abstract}

KEYWORDS: Nanoribbon, nanowire, ion sensitive field effect transistor, $\mathrm{pH}$ sensor, $\mathrm{pH}$ sensing model

\section{Introduction}

Over the past decades, Ion Sensitive Field Effect Transistors (ISFETs) have been intensively researched for a wide range of sensing applications, such as ion [1], pH [2], DNA [3] and protein sensing [4]. More recently, sensors based on silicon nanowires and nanoribbons have also been researched as their high surface-tovolume ratios offer high sensitivity $[5,6]$. Top-down fabricated nanowires [7-10] are compatible with CMOS technology and are therefore preferred to bottom-up nanowires [4, 11], which do not provide precise dimension and position control. However, compared to conventional ISFETs, top-down CMOS nanowires require costly e-beam or deep UV lithography and Silicon-On-Insulator (SOI) substrates [12, 13]. Nanoribbons provide a lower cost alternative to nanowires and can also be fabricated using top-down CMOS technologies [6, 14, 15]. Nanoribbons are typically nanometers thick and a few micrometers wide, thereby eliminating the need for expensive lithography processes. Additionally, thin film transistor (TFT) technologies [16-17] can be employed for nanoribbon 
fabrication and this can further reduce manufacturing cost by eliminating the use of SOI substrates.

Sensing of $\mathrm{pH}$ and proteins is possible when potential changes introduced by analytes on the sensor surface are translated into current changes in the semiconducting channel. For $\mathrm{pH}$ measurements, for example, sensitivities have been reported that vary from $650 \% / \mathrm{pH}$ to $2.5 \% / \mathrm{pH}[18-29]$. A wide variety of technologies were used in these devices, including selfassembled silicon nanowires [23], CMOS-based silicon nanowires fabricated in SOI wafers [20, 25, 26], silicon CMOS-based sheets fabricated in silicon wafers [29] and $\mathrm{MoS}_{2}$ nanoribbons [28]. The highest value of sensitivity was achieved by $\mathrm{MoS}_{2}$ nanoribbons. However, it is unclear whether the excellent sensitivity value obtained for $\mathrm{MoS}_{2}$ nanoribbons is intrinsically due to the use of the 2D material for the biosensor fabrication or due to other aspects of the biosensor design. To date, no explanation has been provided for this wide variation in values of sensitivity.

In this work, we investigate how the transistor performance influences the sensitivity of a wide range of nanowire and nanoribbon sensors. Nanoribbon TFT sensors are fabricated with a broad range of electrical characteristics and values of sensitivity are measured using solutions with different values of $\mathrm{pH}$. A strong relationship between the sensitivity and the device subthreshold slope is experimentally demonstrated. A model based on Bergveld's model [1] and conventional MOSFET theory is then developed which is suitable for benchmarking a broad range of sensors fabricated in different technologies and materials. The model closely matches our experimental results and also explains the wide variation in current sensitivity results reported in the literature.

\section{Experimental procedure}

The sensors used in this work employ in-situ doped polysilicon nanoribbons (Figure 1a) that were manufactured in the three-mask TFT process presented in [17]. Briefly, nanoribbons were fabricated to form junctionless transistors in a single layer of in-situ doped amorphous silicon. A broad range in transistor performance was achieved by varying the dopant concentration in the deposited in-situ doped amorphous silicon layers. Layers with three different doping concentrations were deposited, aimed at low, medium and high doping regimes. The doping concentration was estimated from the measured polysilicon resistance, assuming a field effect mobility of $10 \mathrm{~cm}^{2} / \mathrm{V}$.s and found to be $1 \times 10^{17}, 5 \times 10^{17}$ and $7 \times 10^{18} \mathrm{~cm}-3$ for low, medium and high doped devices respectively. The amorphous silicon layer was subsequently crystallized into polysilicon by annealing. For most devices, the crystallization was performed using a 10 min anneal in dry oxygen at $900^{\circ} \mathrm{C}$ and this also grew $8.5 \mathrm{~nm}$ thermal oxide on top of ribbons. To further diversify the transistor performance, one of the medium doped devices was given an extra (device A) anneal at $600^{\circ} \mathrm{C}$ for 20 hours in nitrogen before the $900^{\circ} \mathrm{C}$ oxidation.

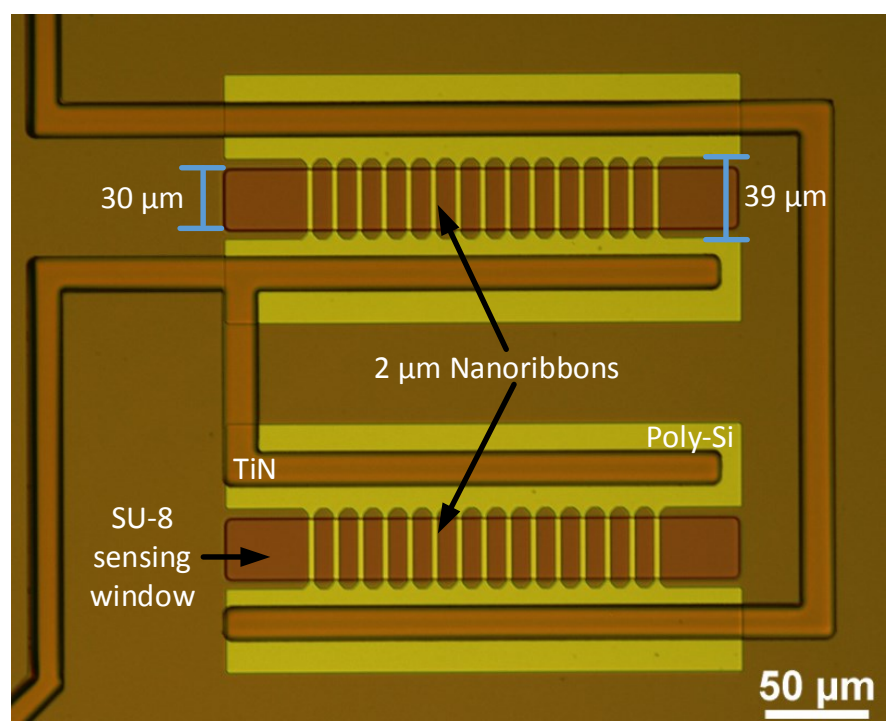

(a)

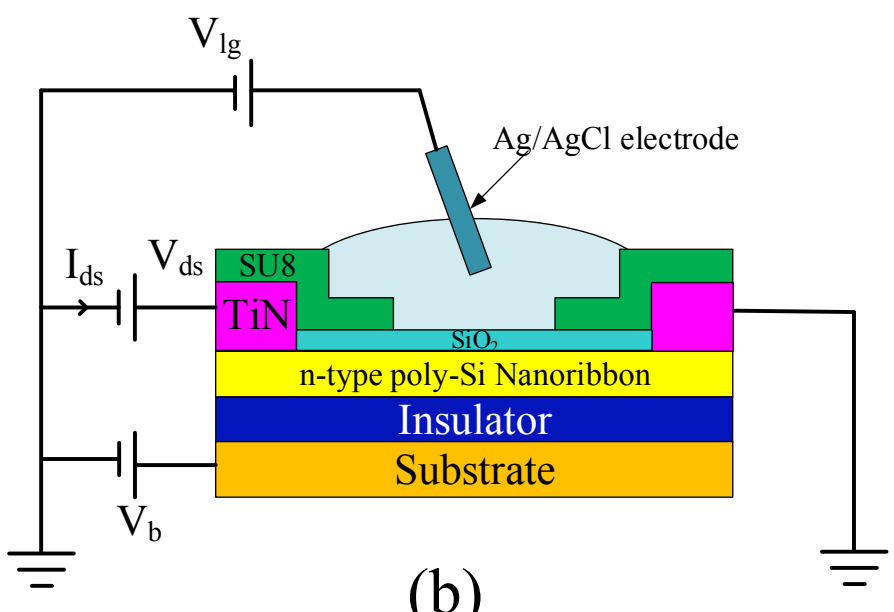

(b)

Figure 1. (a) optical micrograph of the fabricated polysilicon nanoribbon sensor with 30 ribbons in parallel and (b) schematic of the measurement configuration. An external $\mathrm{Ag} / \mathrm{AgCl}$ electrode was used to form a liquid gate and the substrate was used as a back-gate. The device was passivated with SU-8, leaving the ribbons exposed to buffers through the sensing window. 
The above process produces transistors with two gates. The top gate is formed by a liquid gate and an external $\mathrm{Ag} / \mathrm{AgCl}$ electrode, as shown in Figure $1 \mathrm{~b}$. The bottom gate is accessed from the back of the substrate and its voltage is varied (device A) to provide an electrical method of changing the carrier concentration in the polysilicon channel. This provides an additional method of producing transistors with different characteristics.

The $\mathrm{pH}$ sensing measurements were performed using Universal Buffer Mixture (UBM) [19] consisting of $0.1 \mathrm{M} \mathrm{NaCl}, 0.01 \mathrm{M}$ citric acid, $0.01 \mathrm{M}$ phosphoric acid and $0.02 \mathrm{M}$ boric acid adjusted to $\mathrm{pH}$ values ranging from 3 to 9 by titration with a $1 \mathrm{M} \mathrm{NaOH}$ solution. The solutions with $\mathrm{pH}$ between 9 and 3 were pipetting onto the device's sensing window. Washes with deionized (DI) water were used before and after the buffer solution was applied. During $\mathrm{pH}$ sensing, the source was connected to ground and the drain voltage was kept at $100 \mathrm{mV}$. The potential of the liquid gate was controlled through a $\mathrm{Ag} / \mathrm{AgCl}$ electrode, which was swept from -2 to $1 \mathrm{~V}$.

\section{Experimental results}

Figure 2 shows the range of subthreshold characteristics obtained for the fabricated sensors, measured in $\mathrm{pH} 9$ buffer solution, with different channel dopant concentrations and under different values of back-gate bias. The solid lines show subthreshold characteristics for devices with high and medium dopant concentrations, measured at $V_{\mathrm{b}}=0 \mathrm{~V}$. The subthreshold slope improves from 6.2 to $1.4 \mathrm{~V} / \mathrm{dec}$ as the dopant concentration is reduced from $7 \times 10^{18}$ to $5 \times 10^{17} \mathrm{~cm}^{-3}$. Finally the inset shows a subthreshold characteristic for the device with a low dopant concentration. A bottom-gate bias of $7.5 \mathrm{~V}$ was required to turn this device on, as the dopant concentration was low enough to fully deplete the polysilicon layer. The device has a subthreshold slope of $0.23 \mathrm{~V} / \mathrm{dec}$ which is the steepest among our devices. Its superior subthreshold performance can be attributed to its very low doping concentration $\left(1 \times 10^{17} \mathrm{~cm}^{-3}\right)$. This device has on-current of about $6 \mathrm{nA}$, which is much lower than the on-current of the other devices. This is attributed to a high contact resistance and source/drain series resistance, as no highly doped S/D contact was used. The dashed lines show characteristics for the medium doped device (device A) measured at different values of bottom-gate bias, $V_{\mathrm{b}}$. The subthreshold slopes are 1.0, 0.75 and $0.58 \mathrm{~V} / \mathrm{dec}$ at $V_{\mathrm{b}}=0,-10$ and $-20 \mathrm{~V}$ respectively. The subthreshold slope improves with increasing negative bottom-gate bias as the negative bias decreases the carrier concentration in the n-type polysilicon. In summary, devices with a wide range of subthreshold slopes have been successfully produced; the values of subthreshold slope are summarized in Table 1.

Figure 3 shows typical $\mathrm{pH}$ sensing results from sensors with different values of subthreshold slope. The main plots show subthreshold characteristics and the insets show the liquid gate bias as a function of $\mathrm{pH}$ at a reference current, $I_{\text {ref, }}$ in the subthreshold region. Figure $3 \mathrm{a}$ shows $\mathrm{pH}$ sensing results for the device with a subthreshold slope of $0.23 \mathrm{~V} / \mathrm{dec}$ at $\mathrm{pH} 9$.

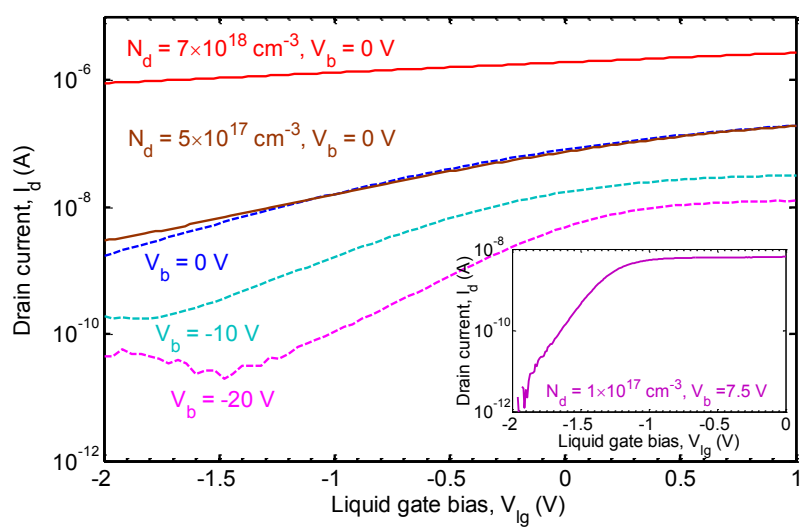

Figure 2. Subthreshold plots of the fabricated devices measured using a $\mathrm{Ag} / \mathrm{AgCl}$ electrode in a liquid gate with a $\mathrm{pH}$ of 9. The solid lines show measurements on devices fabricated with different dopant concentrations, $N_{\mathrm{d}}$, and the dashed lines show measurements on devices at different substrate biases from 0 to $-20 \mathrm{~V}$. The inset shows a subthreshold plot of a device with a low dopant concentration.

The characteristics are parallel in the subthreshold region at different $\mathrm{pH}$ values and therefore, at the reference current, the effect of $\mathrm{pH}$ can be measured as a voltage shift of the liquid gate bias $\left(V_{\mathrm{lg}}\right)$. As shown in the inset, the relation between $V_{\lg }$ and $\mathrm{pH}$ is linear for $\mathrm{pH}$ values between 5 and 9, with a value of 26.8 $\mathrm{mV} / \mathrm{pH}$. Figures $3 \mathrm{~b}$ and $3 \mathrm{c}$ show similar results for the devices with subthreshold slopes of $1.4 \mathrm{~V} / \mathrm{dec}$ and $6.2 \mathrm{~V} / \mathrm{dec}$, respectively. Similar linear trends between liquid gate voltage and $\mathrm{pH}$ are observed and the voltage shifts are extracted to be 30.2 and $26.7 \mathrm{mV} / \mathrm{pH}$ for devices with subthreshold slopes of 1.4 and $6.2 \mathrm{~V} / \mathrm{dec}$, respectively. The extracted values of voltage shift agree very well with the reported voltage shift of $30 \mathrm{mV} / \mathrm{pH}$, for a $\mathrm{SiO}_{2}$ surface [23]. For all three devices, the voltage shifts obtained for $\mathrm{pH} 3$ appear to be outliers. The probable explanation for this result is that the silicon dioxide surface isoelectric point [30] is around 2 and hence it would be expected that the response near $\mathrm{pH} 2$ 
would be smaller because of the lower buffer capability of the $\mathrm{SiO}_{2}$ surface [1].

Table 1 summarizes values of sensitivity for all sensors for comparison with the values of subthreshold slope. The sensitivity is defined as percentage drain current change from $\mathrm{pH} 9$ to $\mathrm{pH} 3$ normalized by the drain current at $\mathrm{pH} 9$ (Sensitivity = $\left.\left(I_{\mathrm{pH} 3}-I_{\mathrm{pH} 9}\right) / I_{\mathrm{pH} 9} \times 100 \%\right)$. As will be explained below, the current sensitivity per $\mathrm{pH}, \mathrm{Sen}_{\mathrm{pH}}$, is extracted from the drain current change from $\mathrm{pH} 9$ to $\mathrm{pH} 5$.
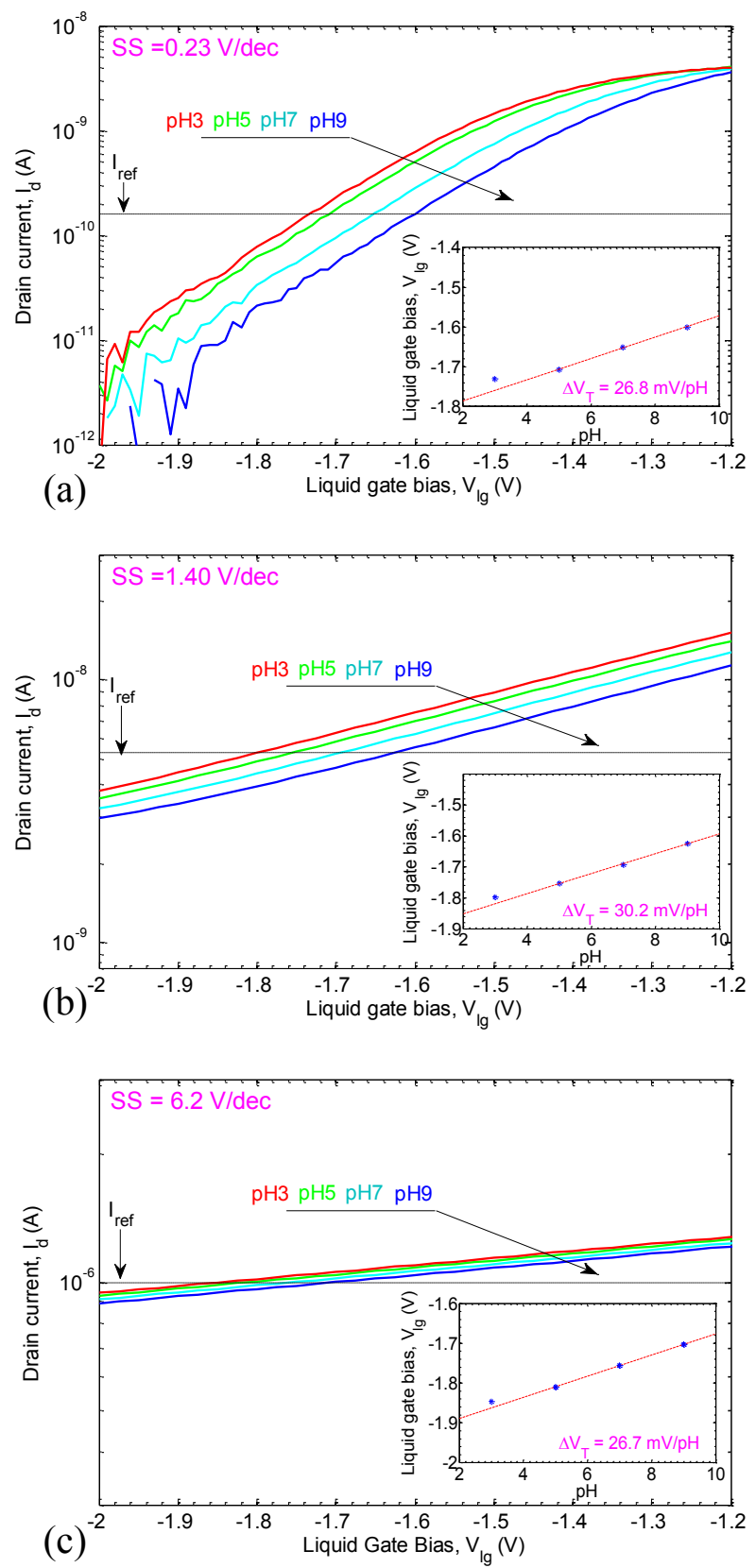

Figure 3. Subthreshold plots showing the effect of $\mathrm{pH}$ on the characteristics of devices with different values of subthreshold slope, $S S$; (a) $S S=0.23 \mathrm{~V} / \mathrm{dec}$, (b) $S S=1.4 \mathrm{~V} / \mathrm{dec}$, and (c) $S S=6.2 \mathrm{~V} / \mathrm{dec}$.
The insets show graphs of liquid gate voltage as a function of $\mathrm{pH}$, measured at reference currents in the subthreshold region, as shown.

The values of subthreshold slope were extracted from the subthreshold characteristics at pH9 (Figure 2). It can be seen that there is a clear correlation between subthreshold slope and sensitivity. The value of sensitivity increases from $7.4 \%$ to $292 \%$ as the subthreshold slope decreases from 6.2 to $0.23 \mathrm{~V} / \mathrm{dec}$.

Table 1. Summary of sensor electrical and $\mathrm{pH}$ sensing results.

\begin{tabular}{ccc}
\hline $\begin{array}{c}\text { Subthreshold slope, } \\
\text { SS (V/dec) }\end{array}$ & $\begin{array}{c}\text { Sensitivity } \\
(\%)\end{array}$ & $\begin{array}{c}\text { Current sensitivity per } \\
\mathrm{pH}, \operatorname{Sen}_{\mathrm{pH}}(\% / \mathrm{pH})\end{array}$ \\
\hline 0.23 & 292 & 33.6 \\
0.58 & 119.7 & 14.0 \\
0.75 & 82.6 & 10.6 \\
1.0 & 55.3 & 7.6 \\
1.4 & 37.0 & 5.9 \\
6.2 & 7.4 & 1.2 \\
\hline
\end{tabular}

\section{Sensitivity model}

To study the relation between sensitivity and device subthreshold slope, a simple general model is proposed. The ISFET operating mechanism derives from sitebinding theory. According to Bergveld's model [1], the electrostatic potential change, $\Delta \Psi_{0}$, due to a $\mathrm{pH}$ change, $\Delta \mathrm{pH}$, at the dielectric surface can be defined as:

$$
\frac{\Delta \psi_{0}}{\Delta p H}=-2.3 \frac{k T}{q} \alpha
$$

with

$$
\alpha=\left(\frac{2.3 k T C_{\text {diff }}}{q^{2} \beta_{\mathrm{int}}}+1\right)^{-1}
$$

where $k$ is the Boltzmann constant, $T$ is the absolute temperature, $q$ is the elementary charge and $\alpha$ is a dimensionless sensitivity parameter between 0 and 1 . The parameter $\alpha$ is dependent on the intrinsic buffer capacity, $\beta_{\text {int, }}$ and the differential capacitance, $C_{\text {diff. }}$ The value of $\beta_{\text {int }}$ depends on the sensing surface binding site density, while $C_{\text {diff }}$ depends on the concentrations and charges of the different ions in the solution. In the ideal case, when the intrinsic buffer capacity is high and the differential capacitance is small, $\alpha$ approaches 1 , leading to a maximum potential change per $\mathrm{pH}$ of $59 \mathrm{mV} / \mathrm{pH}$ at room temperature, which is known as the Nernstian limit. The surface potential change is independent of device dimensions and channel material. 
For a MOSFET, the drain current, $I_{\mathrm{d}}$, in the subthreshold region is expressed as [31]:

$$
\begin{aligned}
& I_{d} \approx \mu_{e f f} C_{o x} \frac{W}{L}(m-1)\left(\frac{k T}{q}\right)^{2} \\
& \exp \left[q\left(V_{l g}-V_{t}\right) / m k T\right]
\end{aligned}
$$

with

$$
S S=\left(\frac{d\left(\log _{10} I_{d}\right)}{d V_{l g}}\right)^{-1}=2.3 \frac{m k T}{q}
$$

where $\mu_{\text {eff }}$ is the effective carrier mobility, $C_{\text {ox }}$ is the top dielectric capacitance, $W$ and $L$ are the width and length of the channel, respectively, $m$ is the body-effect coefficient, $V_{\mathrm{lg}}$ is the liquid gate bias, $V_{\mathrm{t}}$ is the threshold voltage and $S S$ is the subthreshold slope.

For a transistor, the drain current changes exponentially with the gate bias in the subthreshold region and linearly in the linear region. Therefore, sensors are normally operated in the subthreshold region to achieve a large current change [23]. Here, the parasitic resistance effect on sensitivity [18] is not taken into consideration, as the channel resistance in the subthreshold region is much higher than the parasitic resistance. When the $\mathrm{pH}$ value decreases by one unit, from $\mathrm{pH} n$ to $\mathrm{pH}(n-1)$, the oxide surface potential changes by $\Delta \Psi_{0} / \Delta \mathrm{pH}$. This gives a shift in the subthreshold characteristics, which is defined as the liquid gate voltage shift per $\mathrm{pH}, \Delta V_{\mathrm{T}}$. Here, $S S$ is assumed to remain constant between $\mathrm{pH}$ changes, as shown in our experimental results of Figure 3. By substituting $S S$ from Eq. (4) into Eq. (3), the drain current after the $\mathrm{pH}$ change, $I_{\mathrm{d}, \mathrm{pH}(n-1)}$, can be written as:

$$
\begin{array}{r}
\mathrm{I}_{d, p H(n-1)}=\mu_{e f f} \mathrm{C}_{o x} \frac{W}{L}\left(\frac{S S}{2.3} \frac{q}{k T}-1\right)\left(\frac{k T}{q}\right)^{2} \\
\exp \left[2.3\left(\mathrm{~V}_{l g}+\Delta V_{T}-\mathrm{V}_{t}\right) / S S\right] \\
\mathrm{I}_{d, p H(n-1)}=\mu_{e f f} \mathrm{C}_{o x} \frac{W}{L}\left(\frac{S S}{2.3} \frac{q}{k T}-1\right)\left(\frac{k T}{q}\right)^{2} \\
\exp \left[2.3\left(\mathrm{~V}_{l g}-\mathrm{V}_{t}\right) / S S\right] \exp \left(2.3 \Delta V_{T} / S S\right)
\end{array}
$$

In this equation, the $\mathrm{pH}$ change affects only the last exponential term while the other terms remain the same. The unaffected part of Eq. (6) is simply the drain current before the $\mathrm{pH}$ change, $I_{\mathrm{d}, \mathrm{pH} n}$. Therefore, a simple general expression for the current sensitivity per $\mathrm{pH}$, $\mathrm{Sen}_{\mathrm{pH}}$, is obtained by subtracting the drain current before and after the $\mathrm{pH}$ change and dividing by the drain current before:

$$
\begin{aligned}
& \operatorname{Sen}_{p H}=\left(I_{d, p H(n-1)}-I_{d, p H n}\right) / I_{d, p H n} \\
& =\left[\exp \left(2.3 \Delta V_{T} / S S\right)-1\right] \times 100 \%
\end{aligned}
$$

In this expression, the sensitivity is a function of $\Delta V_{\mathrm{T}}$ and the subthreshold slope, $S S$. Here, $\Delta V_{\mathrm{T}}$ is constant for a specific surface and buffer solution as explained above, while $S S$ is determined by the device characteristics.

Using Eq. (7), the current sensitivity per $\mathrm{pH}$ is plotted as a function of $S S$ in Figure 4 for $\Delta V_{\mathrm{T}}=30 \mathrm{mV} / \mathrm{pH}$ and $59 \mathrm{mV} / \mathrm{pH}$. The former value is typical of a $\mathrm{SiO}_{2}$ surface and the latter is typical of $\mathrm{HfO}_{2}$ surface [20] and $\mathrm{Al}_{2} \mathrm{O}_{3}$

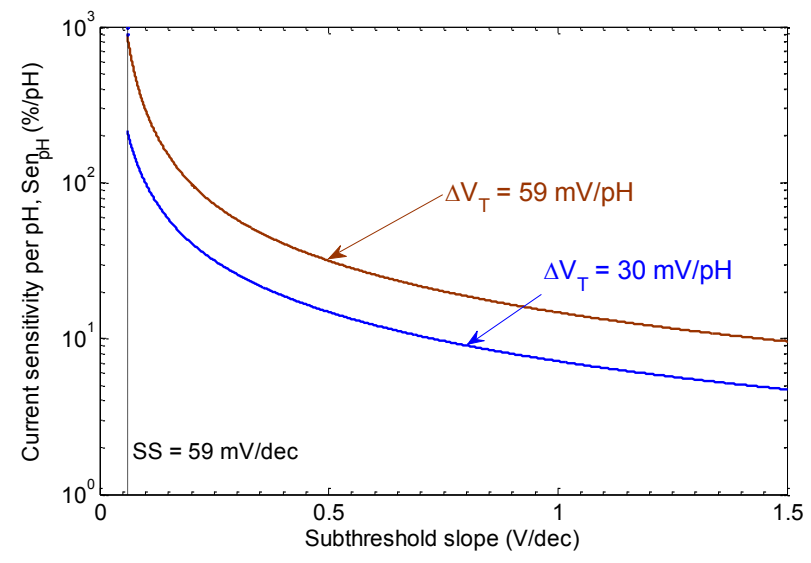

Figure 4. Calculated current sensitivity per $\mathrm{pH}$ (Eq. (7)) as a function of subthreshold slope for two different $\Delta V_{\mathrm{T}}$ values.

surface [19]. Figure 4 shows that the current sensitivity per $\mathrm{pH}$ increases significantly with decreasing subthreshold slope for a given $\Delta V_{\mathrm{T}}$. Thus, a device with a steeper subthreshold slope can deliver a bigger current change during $\mathrm{pH}$ sensing. Furthermore, the use of a dielectric surface like $\mathrm{Al}_{2} \mathrm{O}_{3}$ or $\mathrm{HfO}_{2}$ results in a higher sensitivity when compared to a $\mathrm{SiO}_{2}$ surface, as the surface potential change for the former is significantly bigger than for the latter.

In the literature, $\mathrm{pH}$ sensing results are presented in different forms and in various $\mathrm{pH}$ ranges. To be able to compare these results with our model, the literature sensitivity values need to be converted to current sensitivity per $\mathrm{pH}$. In general, for a $\mathrm{pH}$ change of $m$ units, Eq. (7) can be re-written as: 


$$
I_{d, p H(n-m)}=\left(1+\operatorname{Sen}_{p H}\right)^{m} I_{d, p H n}
$$

where $I_{\mathrm{d}, \mathrm{pH}(n-m)}$ is the drain current change arising from a $\mathrm{pH}$ change of $\mathrm{m}$ units. A more general expression for the current sensitivity per $\mathrm{pH}, \operatorname{Sen}_{\mathrm{pH}}$, can then be derived as:

$$
S e n_{p H}=\left(\sqrt[m]{I_{d, p H(n-m)} / I_{d, p H n}}-1\right) \times 100 \%
$$

This expression can be used to extract values of current sensitivity per $\mathrm{pH}$ from previously published literature.

\section{Discussion}

Figure 5 compares the model with our experimental results (red circles) and with results from the literature (blue symbols for $\mathrm{HfO}_{2}$, green symbols for $\mathrm{Ta}_{2} \mathrm{O}_{5}$, and purple symbols for $\mathrm{SiO}_{2}$ ). The current sensitivity per $\mathrm{pH}$ for the literature work was extracted using Eq. (9) and the $S S$ values were extracted from subthreshold characteristics found in the respective papers. Very good agreement is obtained between our proposed analytical

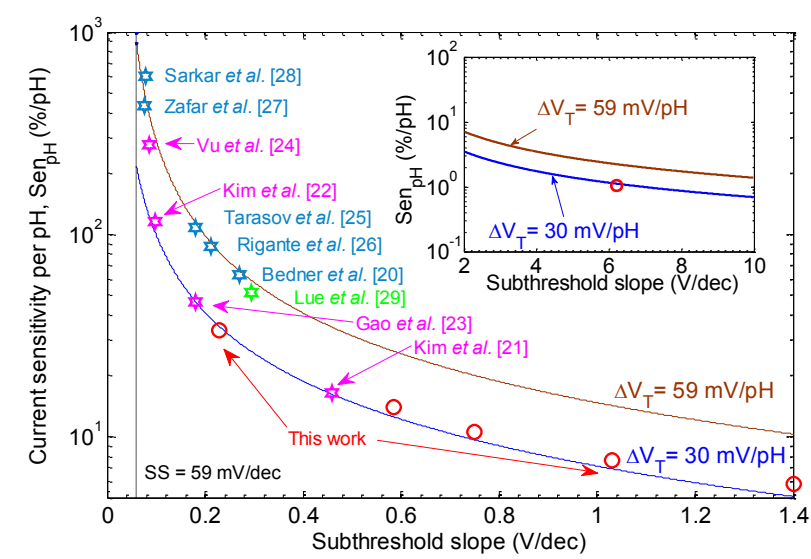

Figure 5. Comparison of calculated sensitivity per pH (Eq. (7)) with our experimental results and with values from the literature. The inset shows the comparison for the device with $S S=6.2 \mathrm{~V} / \mathrm{dec}$.

model and our experimental results. All our experimental data falls close to the calculated curve for $\Delta V_{\mathrm{T}}=30 \mathrm{mV} / \mathrm{pH}$, which is typical for $\mathrm{SiO}_{2}$ surfaces [23]. The experimental results shown in Figure 3 confirm that our $\mathrm{SiO}_{2}$ surface is behaving as expected with $\Delta V_{\mathrm{T}}$ in the range 26.8 to $30.2 \mathrm{mV} / \mathrm{pH}$. Excellent agreement is also obtained with published results for $\mathrm{SiO}_{2}$ sensing surfaces [21-23]. Kim et al. used singlecrystal silicon nanowires with a $\mathrm{SiO}_{2}$ sensing surface and two different $\mathrm{Si}$ thicknesses of $50 \mathrm{~nm}$ [21] and 40 $\mathrm{nm}$ [22]. Gao et al. [23] used bottom-up silicon nanowires with a $\mathrm{SiO}_{2}$ sensing surface. $\mathrm{Vu}$ et al. [24] used top-down silicon nanowires with a $\mathrm{SiO}_{2}$ sensing surface. The data from Vu's work does not agree with the curve of $\Delta V_{\mathrm{T}}=30 \mathrm{mV} / \mathrm{pH}$ in Figure 5. This is attributed to the value of $\Delta V_{\mathrm{T}}$ of $41 \mathrm{mV} / \mathrm{pH}$, which is higher than typically obtained for a typical $\mathrm{SiO}_{2}$ surface.

The brown curve in Figure 5 presents the predicted sensitivity per $\mathrm{pH}$ for $\mathrm{HfO}_{2}$ surfaces and again shows excellent agreement with published results [20, 25-27]. Bedner et al. [20], Tarasov et al. [25] and Rigante et al. [26] used single crystal silicon nanowires with $\mathrm{HfO}_{2}$ sensing surfaces. Zafar et al. [27] used a stacked gate insulator of a $\mathrm{HfO}_{2}$ layer on top of $\mathrm{SiO}_{2}$. The model accurately predicts their sensitivity value, indicating that it can predict the sensitivity of devices with stacked gate insulators as long as the value of $\Delta V_{\mathrm{T}}$ used is for the top dielectric. Sarkar et al. [28] produced $\mathrm{MoS}_{2}$ nanoribbons with a $\mathrm{HfO}_{2}$ sensing surface and achieved a sensitivity of about $600 \% / \mathrm{pH}$. This data also falls on the predicted curve for $\mathrm{HfO}_{2}$ and hence indicates that the model is able to accurately predict the sensitivity of sensors with $\mathrm{MoS}_{2}$, which is a graphene-like material of a few atom layers, as well as single-crystal silicon and polysilicon. Lue et al. [29] used a silicon ISFET with a stacked gate insulator of $\mathrm{Ta}_{2} \mathrm{O}_{5}$ layer on top of $\mathrm{SiO}_{2}$. The result slightly falls off the predicted sensitivity curve of $\Delta V_{\mathrm{T}}=59 \mathrm{mV} / \mathrm{pH}$ as the $\mathrm{Ta}_{2} \mathrm{O}_{5}$ layer was not optimized with a $\Delta V_{\mathrm{T}}$ of $51.8 \mathrm{mV} / \mathrm{pH}$.

The results in Figure 5 indicate that current sensitivity per $\mathrm{pH}$ is directly related to the subthreshold slope of the sensing transistor. It is widely accepted that a high 'surface-to-volume' ratio improves the sensitivity and there has therefore been a tendency in the literature to use aggressive scaling of the sensing transistor by employing nanowires, nanoribbons, FinFETs and 2-D transistors. However, our results show that aggressive scaling is not the key to higher sensitivity, but rather the design of a sensing transistor with a good value of subthreshold slope. Furthermore, Figure 5 shows that reasonable values of sensitivity per $\mathrm{pH}$ can be achieved using low-cost thin film technology that is ideally suited for mass production.

The results in Figure 5 also indicate that sensitivity per $\mathrm{pH}$ is a useful figure-of-merit for comparing the performance of sensors fabricated in a wide range of different materials. The material itself only affects the sensor performance through the value of subthreshold slope that can be achieved with transistors fabricated in that material. If we consider an ideal device with a subthreshold slope, $S S$, of $59 \mathrm{mV} / \mathrm{dec}$ the model predicts that the highest achievable value of current sensitivity per $\mathrm{pH}$ is $222 \% / \mathrm{pH}$ for a $\mathrm{SiO}_{2}$ sensing surface and 
$900 \% / \mathrm{pH}$ for a $\mathrm{HfO}_{2}$ surface regardless of the material that the ribbons or nanowires are made of. Current sensitivity per $\mathrm{pH}$ is also a good benchmarking figureof-merit for comparing nanowire and nanoribbon sensors. Nanowire sensors will only give better values of sensitivity than nanoribbon sensors if they have a better value of subthreshold slope.

Signal-to-Noise ratio (SNR) is an important factor that together with the sensitivity, defines the limit of detection for biosensors [32, 33]. SNR was reported to be mainly affected by the intrinsic device quality [32] and to increase with the square root of channel area [33]. In our work, it has been shown that aggressive scaling is not necessary to achieve high sensitivity and therefore devices such as nanoribbons with large channel areas can be used to deliver high sensitivity without compromising the signal-to-noise ratio. It can therefore be concluded that the model presented in this work provides a useful figure-of-merit for comparing the performance of sensors fabricated using different methods and fabricated in different materials.

\section{Conclusions}

In this work, we have fabricated nanoribbon sensors with a range of subthreshold slopes to investigate the effect of subthreshold slope on the sensitivity of nanoribbon $\mathrm{pH}$ sensors. The results show a strong correlation between the sensitivity and the subthreshold slope, with the sensitivity increasing with decreasing subthreshold slope. A simple model is developed, which depends only on the subthreshold slope of the sensor and the sensing surface. The model accurately predicts our experimental results as well as literature results for nanowire and nanoribbon sensors formed using different sensing surfaces, such as $\mathrm{SiO}_{2}$ and $\mathrm{HfO}_{2}$, and different semiconductor materials, such as single-crystal $\mathrm{Si}$, polysilicon and $\mathrm{MoS}_{2}$. The model defines sensor sensitivity as current sensitivity per $\mathrm{pH}$, thereby allowing this figure-of-merit to be used for benchmarking the performance of sensors fabricated using different methods or materials. In this way, the model provides a clear route for optimizing the performance of nanoFET sensor technologies.

\section{Acknowledgments}

The Authors declare no competing financial interest. The authors would like to acknowledge the Technology Strategy Board (TSB) and the Engineering and Physical Sciences Research Council (EPSRC: EP/K502327/1) for funding this work. We would also like to thank Sally
Anderson, Gregory Gay, Ben Hadwen and Chris J. Brown of Sharp Laboratories Europe for many useful discussions. The data for this paper can be found at $10.5258 / \mathrm{SOTON} / 380117$.

\section{References}

[1] P. Bergveld, "Thirty years of ISFETOLOGY What happened in the past 30 years and what may happen in the next 30 years," Sensors and Actuators B-Chemical, vol. 88, pp. 1-20, Jan 1 2003.

[2] J. Bausells, J. Carrabina, A. Errachid, and A. Merlos, "Ion-sensitive field-effect transistors fabricated in a commercial CMOS technology," Sensors and Actuators B-Chemical, vol. 57, pp. 56-62, Sep 71999.

[3] E. Stern, J. F. Klemic, D. A. Routenberg, P. N. Wyrembak, D. B. Turner-Evans, A. D. Hamilton, et al., "Label-free immunodetection with CMOS-compatible semiconducting nanowires," Nature, vol. 445, pp. 519-522, Feb 12007.

[4] G. F. Zheng, F. Patolsky, Y. Cui, W. U. Wang, and C. M. Lieber, "Multiplexed electrical detection of cancer markers with nanowire sensor arrays," Nature Biotechnology, vol. 23, pp. 1294-1301, Oct 2005.

[5] M. M. A. Hakim, M. Lombardini, K. Sun, F. Giustiniano, P. L. Roach, D. E. Davies, et al., "Thin Film Polycrystalline Silicon Nanowire Biosensors," Nano Letters, vol. 12, pp. 18681872, Apr 2012.

[6] X. X. Duan, Y. Li, N. K. Rajan, D. A. Routenberg, Y. Modis, and M. A. Reed, "Quantification of the affinities and kinetics of protein interactions using silicon nanowire biosensors," Nature Nanotechnology, vol. 7, pp. 401-407, Jun 2012.

[7] B. R. Dorvel, B. Reddy, J. Go, C. D. Guevara, E. Salm, M. A. Alam, et al., "Silicon Nanowires with High-k Hafnium Oxide Dielectrics for Sensitive Detection of Small Nucleic Acid Oligomers," Acs Nano, vol. 6, pp. 6150-6164, Jul 2012.

[8] Z. Li, Y. Chen, X. Li, T. I. Kamins, K. Nauka, and R. S. Williams, "Sequence-specific label-free DNA sensors based on silicon nanowires," Nano Letters, vol. 4, pp. 245-247, Feb 2004. 
[9] A. Tarasov, M. Wipf, K. Bedner, J. Kurz, W. Fu, V. A. Guzenko, et al., "True Reference Nanosensor Realized with Silicon Nanowires," Langmuir, vol. 28, pp. 9899-9905, Jun 262012.

[10] G. J. Zhang, L. Zhang, M. J. Huang, Z. H. H. Luo, G. K. I. Tay, E. J. A. Lim, et al., "Silicon nanowire biosensor for highly sensitive and rapid detection of Dengue virus," Sensors and Actuators B-Chemical, vol. 146, pp. 138-144, Apr 82010.

[11] Y. Cui, Q. Q. Wei, H. K. Park, and C. M. Lieber, "Nanowire nanosensors for highly sensitive and selective detection of biological and chemical species," Science, vol. 293, pp. 1289-1292, Aug 172001.

[12] Y. L. Bunimovich, Y. S. Shin, W. S. Yeo, M. Amori, G. Kwong, and J. R. Heath, "Quantitative real-time measurements of DNA hybridization with alkylated nonoxidized silicon nanowires in electrolyte solution," Journal of the American Chemical Society, vol. 128, pp. 16323-16331, Dec 202006.

[13] J. H. Chua, R. E. Chee, A. Agarwal, S. M. Wong, and G. J. Zhang, "Label-Free Electrical Detection of Cardiac Biomarker with Complementary Metal-Oxide Semiconductor-Compatible Silicon Nanowire Sensor Arrays," Analytical Chemistry, vol. 81, pp. 6266-6271, Aug 12009.

[14] N. Elfstrom, A. E. Karlstrom, and J. Linnros, "Silicon nanoribbons for electrical detection of biomolecules," Nano Letters, vol. 8, pp. 945949, Mar 2008.

[15] E. Stern, A. Vacic, N. K. Rajan, J. M. Criscione, J. Park, B. R. Ilic, et al., "Label-free biomarker detection from whole blood," Nature Nanotechnology, vol. 5, pp. 138-142, Feb 2010.

[16] Y. L. Wu, P. Y. Hsu, C. P. Hsu, and W. C. Liu, "Polysilicon Wire for the Detection of LabelFree DNA," Journal of the Electrochemical Society, vol. 157, pp. J191-J195, 2010.

[17] K. Sun, I. Zeimpekis, M. Lombardini, N. M. J. Ditshego, S. J. Pearce, K. S. Kiang, et al., "ThreeMask Polysilicon Thin-Film Transistor Biosensor," leee Transactions on Electron Devices, vol. 61, pp. 2170-2176, Jun 2014.

[18] I. Zeimpekis, K. Sun, C. X. Hu, O. Thomas, M. R. R. de Planque, H. M. H. Chong, et al., "Study of parasitic resistance effects in nanowire and nanoribbon biosensors," Nanoscale Research Letters, vol. 10, Feb 212015.

[19] S. Y. Chen, J. G. Bomer, E. T. Carlen, and A. van den Berg, "Al2O3/Silicon NanolSFET with Near Ideal Nernstian Response," Nano Letters, vol. 11, pp. 2334-2341, Jun 2011.

[20] K. Bedner, V. A. Guzenko, A. Tarasov, M. Wipf, R. L. Stoop, D. Just, et al., "pH Response of Silicon Nanowire Sensors: Impact of Nanowire Width and Gate Oxide," Sensors and Materials, vol. 25, pp. 567-576, 2013.

[21] S. Kim, K. Kim, T. Rim, C. Park, D. Cho, C. K. Baek, et al., "pH sensing and noise characteristics of Si nanowire ion-sensitive field effect transistors," in Nano/Micro Engineered and Molecular Systems (NEMS), 2011 IEEE International Conference on, 2011, pp. 12331236.

[22] S. Kim, T. Rim, K. Kim, U. Lee, E. Baek, H. Lee, et al., "Silicon nanowire ion sensitive field effect transistor with integrated $\mathrm{Ag} / \mathrm{AgCl}$ electrode: $\mathrm{pH}$ sensing and noise characteristics," Analyst, vol. 136, pp. 5012-5016, 2011.

[23] X. P. A. Gao, G. F. Zheng, and C. M. Lieber, "Subthreshold Regime has the Optimal Sensitivity for Nanowire FET Biosensors," Nano Letters, vol. 10, pp. 547-552, Feb 2010.

[24] X. T. Vu, J. F. Eschermann, R. Stockmann, R. GhoshMoulick, A. Offenhausser, and S. Ingebrandt, "Top-down processed silicon nanowire transistor arrays for biosensing," Physica Status Solidi a-Applications and Materials Science, vol. 206, pp. 426-434, Mar 2009.

[25] A. Tarasov, "Silicon Nanowire Field-Effect Transistors for Sensing Applications," PhD, University of Basel, 2012.

[26] S. Rigante, M. Wipf, A. Bazigos, K. Bedner, D. Bouvet, and A. M. lonescu, "Finfet with Fully pH-Responsive $\mathrm{HfO}_{2}$ as Highly Stable Biochemical Sensor," 2014 leee 27th International Conference on Micro Electro Mechanical Systems (MEMS), pp. 1063-1066, 2014.

[27] S. Zafar, C. D'Emic, A. Afzali, B. Fletcher, Y. Zhu, and $\mathrm{T}$. Ning, "Optimization of $\mathrm{pH}$ sensing using silicon nanowire field effect transistors with 
HfO2 as the sensing surface," Nanotechnology, vol. 22, pp. 405501, Oct 72011.

[28] D. Sarkar, W. Liu, X. J. Xie, A. C. Anselmo, S. Mitragotri, and K. Banerjee, "MoS2 Field-Effect Transistor for Next-Generation Label-Free Biosensors," Acs Nano, vol. 8, pp. 3992-4003, Apr 2014.

[29] C. E. Lue, T. C. Yu, C. M. Yang, D. G. Pijanowska, and C. S. Lai, "Optimization of Urea-EnFET Based on Ta2O5 Layer with Post Annealing," Sensors, vol. 11, pp. 4562-4571, May 2011.

[30] L. Bousse and S. Mostarshed, "The ZetaPotential of Silicon-Nitride Thin-Films," Journal of Electroanalytical Chemistry, vol. 302, pp. 269-274, Mar 111991.

[31] Y. Taur and T. H. Ning, Fundamentals of modern VLSI devices, 2nd ed. Cambridge, UK ; New York: Cambridge University Press, 2009.

[32] N. K. Rajan, D. A. Routenberg, and M. A. Reed, "Optimal signal-to-noise ratio for silicon nanowire biochemical sensors," Applied Physics Letters, vol. 98, pp. 264107, Jun 272011.

[33] N. K. Rajan, K. Brower, X. X. Duan, and M. A. Reed, "Limit of detection of field effect transistor biosensors: Effects of surface modification and size dependence," Applied Physics Letters, vol. 104, pp. 084106, Feb 24 2014. 